\section{Neues Kriterium in der Asthmatherapie?}

Fuhlbrigge AL et al. A novel endpoint for exacerbations in asthma to accelerate clinical development: a post-hoc analysis of randomised controlled trials. Lancet Respir Med 2017; 5 : $577-590$

Exazerbationen bei Asthma bronchiale sind von den Betroffenen besonders gefürchtet. Daher ist es vorrangiges Ziel der Therapie, diese zu vermeiden. Definiert als schwere Luftnot bei steroidpflichtigem Asthma, die eine Notfalltherapie erfordert, sind Exazerbationen selten. Studien mit diesem Endpunkt müssen daher sehr viele Patienten umfassen, um überhaupt zu Ergebnissen zu kommen. Ein alternativer Endpunkt, der auch kleinere aussagekräftige Studien ermöglichen würde, wäre also wünschenswert.

Ziel der Autoren war es, einen Endpunkt zu etablieren, der verschiedene Parameter erfasst, anhand derer sich das Risiko für eine Exazerbation vorhersagen lässt. Dieser Endpunkt soll es ermöglichen, kleinere Studien durchzuführen, die trotzdem Aussagen dazu erlauben, ob ein neues Medikament den Patienten vor Exazerbationen schützen kann. Die Autoren werteten 12 randomisierte Studien mit Asthmatikern aus, bei denen neben den Exazerbationen verschiedene andere Parameter dokumentiert wurden. In den Studien waren verschiedene Präparate über jeweils 6-12 Monate verglichen worden. Die mehr als 17.000 Patienten im mittleren Alter von 35 bis 53 Jahren, überwiegend weiblich, wiesen einen FEV1 von 63-84\% des Sollwerts auf. Anhand der Daten von 5 Studien entwickelten die Autoren einen Parameter namens CompEx. Ausgehend von den Befunden Peak expiratory Flow (P), Gebrauch eines Relievers (R), Wachwerden wegen Beschwerden (A) und Symptomen (S) prüften die Autoren verschiedene Kombinationen (z.B. PR, PS oder PRSA). Die möglichen Kombinationen wurden in weiteren 7 Studien auf ihre Aussagekraft hin überprüft. Schließlich definierten Fuhlbrigge et al. einen Endpunkt CompEx, basierend auf PRS (definiert als erstmaliges Ereignis im Asthmatagebuch) oder Exazerbation. Als relevantes Ereignis im Tagebuch galt eine anhand bestimmter Grenzwerte und Veränderung über die Zeit (slope) definierte deutliche Verschlechterung der Parameter $\mathrm{P}, \mathrm{R}$ oder $\mathrm{S}$.
Anschließend verglichen sie die Aussagekraft von CompEx hinsichtlich Therapieeffekt und benötigter Studiengröße im Vergleich zum Endpunkt „schwere Exazerbation“. Würde als Endpunkt CompEx genutzt, würde dies im Vergleich zum Endpunkt Exazerbation zu 2,8-mal häufigeren Ereignissen führen, gleichzeitig ergab sich für den Therapieeffekt das gleiche Ergebnis.

\section{FAZIT}

Dieser neu definierte Endpunkt CompEx zur Bewertung von Effekten neuer Therapien bei Asthma bronchiale würde im Vergleich zum Endpunkt Exazerbation kürzere Studien mit über $50 \%$ weniger Patienten erlauben, die dennoch eine ebenso hohe Aussagekraft hätten, schreiben die Autoren. So ließe sich vermeiden, unnötig viele Patienten in Studien einem eventuell wirkungslosen Präparat auszusetzen.

Dr. med. Susanne Meinrenken, Bremen 